\title{
Repeated Combined Chemotherapy with Cisplatin Lowers Carnitine Levels in Gastric Cancer Patients
}

\author{
Akimasa Kawai $^{a}$ Hideo Matsumoto ${ }^{a}$ Youko Endou $^{b}$ Yui Honda ${ }^{b}$ \\ Hisako Kubota $^{c}$ Masaharu Higashida ${ }^{c}$ Toshihiro Hirai $^{d}$ \\ a Department of Surgery, Mitsugi Public General Hospital, Onomichi, Japan; b Department of Nutrition, Kawasaki \\ Medical School Hospital, Japan, Kurashiki, Japan; ' Department of Digestive Surgery, Kawasaki Medical School, \\ Kurashiki, Japan; ' Department of Clinical Nutrition, Kawasaki University of Medical Welfare, Kurashiki, Japan
}

\author{
Keywords \\ Carnitine $\cdot$ Chemotherapy $\cdot$ Ciplatin $\cdot$ Nutrition . \\ Gastric cancer
}

\begin{abstract}
Background/Aims: Carnitine plays an important role in the metabolism of fatty acids. It has also been reported that the administration of anticancer drugs may lead to reductions in serum carnitine levels due to decreased activity of organic cation transporter novel 2, which plays a role in the reabsorption of carnitine in the tubules of the kidney. We therefore studied the change in carnitine levels when chemotherapy was administered repeatedly to patients with gastric cancer. Methods: Ten patients with upper gastrointestinal cancer were enrolled in this study between December 2014 and August 2015. All patients were administered chemotherapy consisting of TS- 1 and cisplatin every 3 weeks: 3 received it as adjuvant therapy post resection, the remaining 7 received it as treatment for unresectable tumors. Before the start of each chemotherapy cycle, serum was collected. Results: The mean total carnitine level was $54.5 \pm 13.7$ $\mu \mathrm{mol} / \mathrm{L}$ prior to commencing chemotherapy; it was $46.7 \pm$ 13.5 and $41.4 \pm 14.8 \mu \mathrm{mol} / \mathrm{L}$ at the second and third cycles
\end{abstract}

respectively. The total carnitine level was decreased in a statistically significant manner $(p=0.0039)$. The serum level of total protein and cholinesterase was also decreased significantly ( $p=0.0218$ and $p=0.0418$ ). Conclusion: Carnitine levels decreased during repeated chemotherapy in patients with gastric cancer, and they are associated with the nutritional status.

(c) 2017 S. Karger AG, Basel

\section{Introduction}

Carnitine is a vitamin-like substance present in trace amounts, synthesized from methionine and lysine in the kidney, liver, and brain [1]. It plays an important role in the metabolism of fatty acids [2].

Deficiencies of carnitine are reported to cause severe symptoms such as muscle weakness, fatigue, heart failure, and hyperammonemia [3, 4]. Furthermore, carnitine deficiencies have been reportedly associated with subjective symptoms in cancer patients $[5,6]$.

It has also been reported that the administration of anticancer drugs may lead to reductions in serum carnitine levels due to decreases in the activity of the organic cation

\section{KARGER}

(C) 2017 S. Karger AG, Basel

E-Mail karger@karger.com

www.karger.com/anm
Akimasa Kawai

Department of Surgery, Mitsugi General Hospital

124 Ichi Mitsugi Town

Onomichi City, Hiroshima 722-0393 (Japan)

E-Mail aki.gi.since2012@gmail.com 


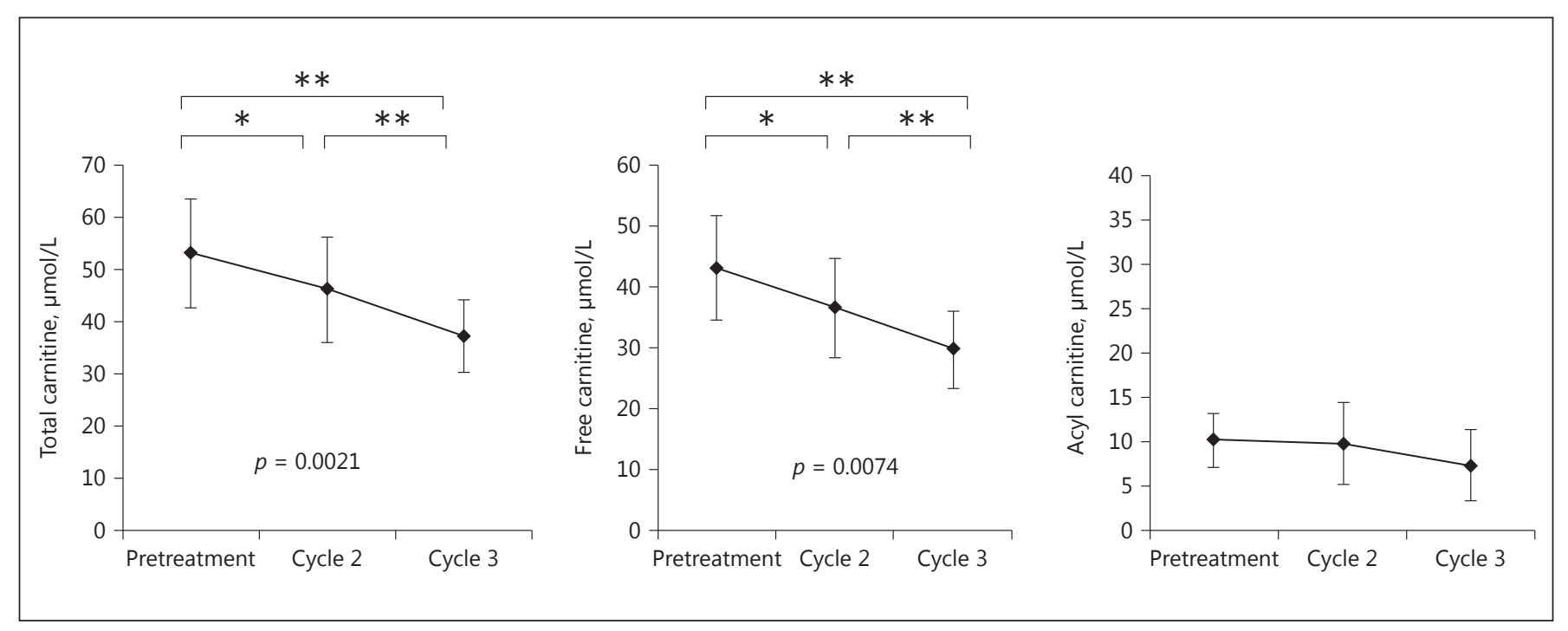

Fig. 1. Changes in serum carnitine level. ${ }^{*} p<0.05 ;{ }^{* *} p<0.01$.

transporter novel 2, which plays a role in reabsorption of carnitine in the tubules of the kidneys [7].

We therefore assumed that repeated chemotherapy might reduce carnitine levels significantly and be associated with nutritional disorders.

\section{Patients and Methods}

This study was proposed prospectively. Ten sequential patients (5 men, 5 women) with gastric cancer were enrolled in this study between December 2014 and August 2015. All patients were administered chemotherapy consisting of TS-1 $\left(80 \mathrm{mg} / \mathrm{m}^{2}\right)$ and cisplatin $\left(60 \mathrm{mg} / \mathrm{m}^{2}\right)$ every 3 weeks as they were all at stage IV of the disease. Three patients (women) received gastrectomy in stage IIIC and 7 patients were unresectable gastric cancer patients in stage IV according to UICC TNM classification. One day before the start of each chemotherapy cycle, serum was collected and analyzed for the level of total protein (TP), albumin (Alb), cholinesterase, and total cholesterol at the laboratory of Kawasaki Medical School Hospital. The level of total carnitine, acyl-carnitine, and free-carnitine was analyzed at an outsourced laboratory company (SRL Inc., Tokyo, Japan). Normal lower limits of total, free, and acyl carnitine level were more than $45.9,36.7$, and $6.2 \mu \mathrm{mol} / \mathrm{L}$, respectively. The percentage of body muscle mass was measured using bioelectrical impedance analysis by a Body Water Analyzer (In Body S10, In Body Japan, Tokyo, Japan).

When the serum carnitine level decreased below the low normal range in this study, l-carnitine was administered as soon as the result was known.

This study protocol was approved by the Institutional Review Board of Kawasaki

Medical School (Approval No. 1855) and informed consent was obtained from all patients.

\section{Statistical Analysis}

Correlations between groups were determined by the chisquare test. All statistical calculations were performed with JMP ${ }^{\circledR}$ 10 software (SAS Institute Inc., Cary, NC, USA). A $p$ value $<0.05$ was considered to be statistically significant.

\section{Results}

Three female patients received chemotherapy of TS-1 + cisplatin after gastrectomy, while the other 7 patients received it as treatment for their unresectable cancer. All patients received more than 2 cycles. The mean age was 63.8 years. Five patients were able to undergo evaluation for more than 4 cycles.

\section{Carnitine}

Total carnitine and free-carnitine levels were significantly reduced from 53.2 and 42.9 to 37.2 and $29.8 \mu \mathrm{mol} / \mathrm{L}$ ( $p=0.0021$ and $p=0.0074)$, respectively, at the beginning of chemotherapy and before the third cycle. The acyl-carnitine level also decreased from 10.3 to $7.4 \mu \mathrm{mol} / \mathrm{L}$ but did not reach statistical significance (Fig. 1). The level of carnitine in 4 patients decreased below the accepted lower limit; therefore, they were administered l-carnitine (l-cartin ${ }^{\circledR}$ FF tablet, Otsuka, Japan) after 2 cycles in this study. Five patients could be treated for more than 4 cycles without administration of the l-carnitine. 


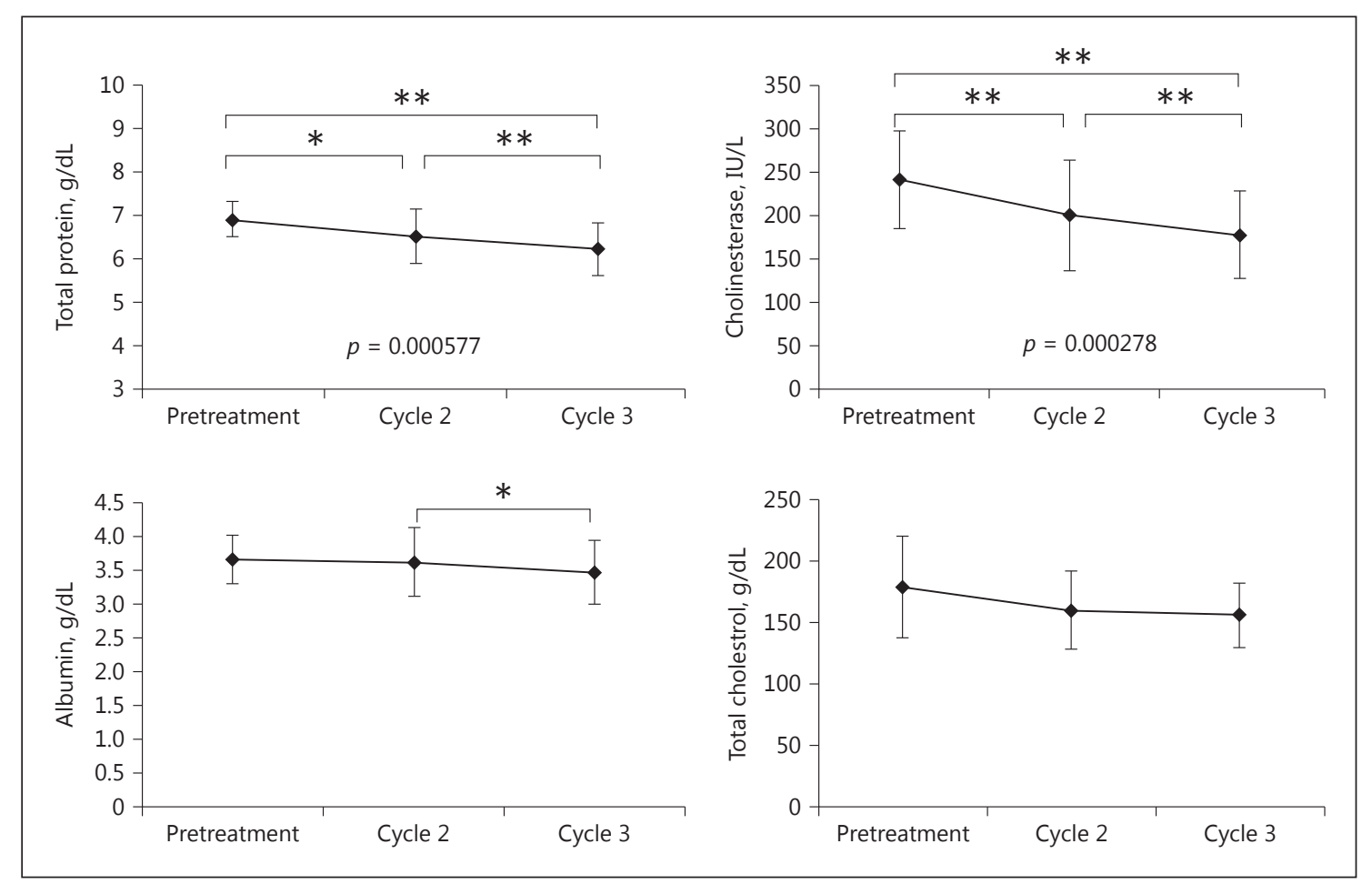

Fig. 2. Changes in nutritional status. ${ }^{*} p<0.05$; ${ }^{* *} p<0.01$.

\section{Nutritional Status}

The mean serum level of TP and cholinesterase was significantly decreased from $6.9 \mathrm{~g} / \mathrm{dL}$ and $241.4 \mathrm{IU} / \mathrm{L}$ to $6.23 \mathrm{~g} / \mathrm{dL}$ and $178.1 \mathrm{IU} / \mathrm{L}$, respectively $(p=0.000577$ and $p=0.00278$ ). The mean serum level of Alb and total cholesterol was decreased from $3.7 \mathrm{~g} / \mathrm{dL}$ and $167.9 \mathrm{IU} / \mathrm{L}$ to $3.53 \mathrm{~g} / \mathrm{dL}$ and $157.3 \mathrm{IU} / \mathrm{L}$ respectively. However, there was no significant difference between them (Fig. 2).

\section{Body Measurements by a Body Water Analyzer}

The mean value of the body mass index and body fat percentage was significantly decreased from $21.9 \mathrm{~kg} / \mathrm{m}^{2}$ and $25.0 \%$ to $21.1 \mathrm{~kg} / \mathrm{m}^{2}$ and $22.7 \%$ respectively. The mean value of skeletal muscle mass and visceral fat area percentage was not changed (Fig. 3).

\section{Discussion}

It has been reported that the concentration of carnitine after chemotherapy is decreased after chemotherapy, especially using platinum-based anticancer drugs.
Impaired expression of organic cation transporter novel 2 , the principle carnitine transporter, in proximal tubular cells suppresses the reabsorption of carnitine and the urinary loss of carnitine was increased [7]. Ikezaki et al. [8] reported that the concentration of urinary carnitine levels differed significantly on days 2 and 3 after chemotherapy and were associated with self-reported fatigue. Conversely, Gomi et al. [9] reported that the change in plasma and urinary carnitine levels was not related to clinical factors.

These reports were based on the result of measurements of serum carnitine levels for one cycle of chemotherapy. The level of carnitine decreased below the low normal range after 2 cycles of treatment. Four patients were administered 1 -carnitine as soon as the results were known; however, time differences existed in the results obtained because the examination was performed by an outside institution. The chemotherapy for all of them was continued by administering l-carnitine.

Interestingly, the serum levels of TP, Alb, and ChoE had decreased significantly and appeared to be synchronized with the level of carnitine. It was thought that the adverse effects such as appetite loss and/or nausea caused by chemotherapy led to the deterioration of the nutritional status. 


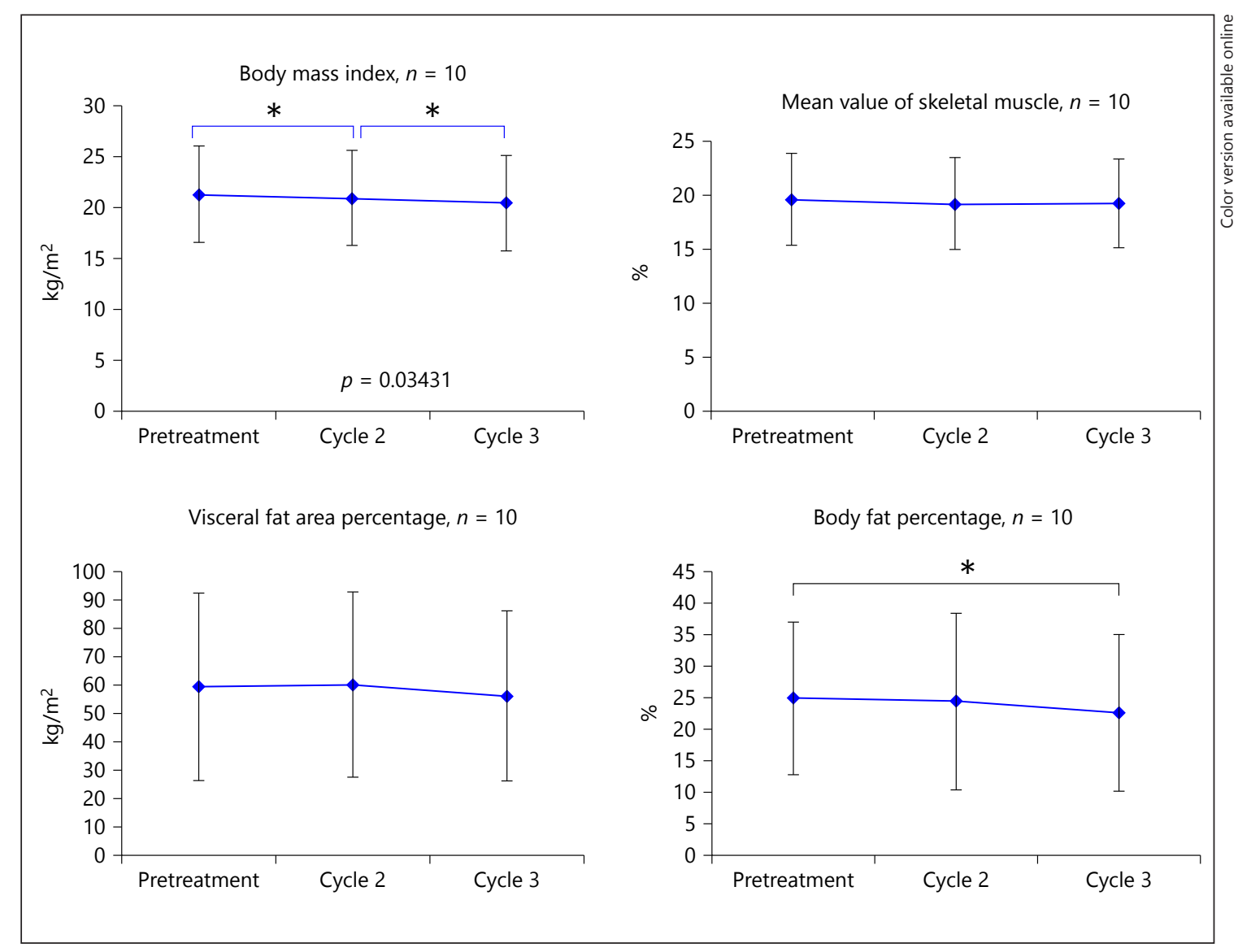

Fig. 3. Changes in body measurements. ${ }^{*} p<0.05$.

Second, decreasing the serum level of carnitine might cause a deterioration in the serum Alb. It was reported that plasma carnitine levels correlated positively with serum Alb in patients on prolonged total parenteral nutrition [10]. Furthermore, there were many reports that supplementation of carnitine improved the nutritional status in hepatocellular carcinoma patients [11, 12] and hemodialysis patients [13]. Muto et al. [14] reported that carnitine deficiency inhibits fatty acid mobilization and oxidation for energy in skeletal muscles. Skeletal muscles are presumed to utilize branched-chain amino acids as a substitute for fatty acids to produce energy in liver cirrhosis patients. The decrease in Alb levels might be caused by this mechanism.

In our study, mean values of body mass index and body fat percentages were significantly decreased. However, the mean value of skeletal muscle mass and visceral fat area was not changed. That might be due to the short observation period at 2 cycles ( 6 weeks).
Three patients whose carnitine levels were below the low normal range were administered l-cartine and continued chemotherapy, while this regimen was discontinued in one patient. Five patients completed more than 4 cycles of chemotherapy.

These data suggest that the supplementation of carnitine for platinum-based chemotherapy might be effective in order to complete the chemotherapy regimen and to improve the quality of life of patients. We are planning the next trial if the supplementation of carnitine might indeed improve nutritional status and quality of life.

This study did have several limitations. This study was conducted at a single institute and the number of patients enrolled was small. Also, this study did not prove the relationship of the mechanism between carnitine levels and Alb.

In conclusion, carnitine levels might decrease in response to chemotherapy and some patients might need supplemental carnitine during such repeated chemotherapy regimens. 


\section{Acknowledgments}

This research study was conducted using Kawasaki Medical School's faculty research expenses. We would like to thank Editage (www.editage.jp) for their English language editing services.

This manuscript has not been published elsewhere and it has not been submitted simultaneously for publication elsewhere.

\section{Disclosure Statement}

The authors have no conflicts of interest to declare.

\section{References}

$>1$ Wanner C, Hörl WH: Carnitine abnormalities in patients with renal insufficiency. Pathophysiological and therapeutical aspects. Nephron 1988;50:89-102.

$\checkmark 2$ Fritz IB, Marquis NR: The role of acylcarnitine esters and carnitine palmityltransferase in the transport of fatty acyl groups across mitochondrial membranes. Proc Natl Acad Sci U S A 1965;54:1226-1233.

$\checkmark 3$ Pons R, De Vivo DC: Primary and secondary carnitine deficiency syndrome. J Clin Neurol 1995; 10:S8-S24.

4 Editorials: Carnitine deficiency. Lancet 1990; 335:981-982.

5 Costanzo M, Cannizzaro MA: Decrease of serum carnitine levels in patients with or without gastrointestinal cancer cachexia. World J Gastroenterol 2006;12:4541-4545.

-6 Busquets S, Serpe R, Toledo M, Betancourt A, Marmonti E, Orpí M, Pin F, Capdevila E, Madeddu C, López-Soriano FJ, Mantovani G, Macciò A, Argilés JM: L-carnitine: an adequate supplement for a multi-targeted anti-wasting therapy in cancer. Clin Nutr 201;31:889-895.

7 Haschke M, Vitins T, Lüde S, Todesco L, Novakova K, Herrmann R, Krähenbühl S: Uri- nary excretion of carnitine as a marker of proximal tubular damage associated with platin-based antineoplastic drugs. Nephrol Dial Transplant 2010;25:426-433.

8 Ikezaki T, Suzuki K, Kambara K, Inomata M, Okazawa S, Kajiura S, Miwa T, Tanabe K, Kashii T: Relationship between carnitine pharmacokinetics and fatigue in patients treated with cisplatin-containing chemotherapy. Oncol Res Treat 2017;40:42-45.

9 Gomi D, Tanaka A, Fukushima T, Kobayashi T, Matsushita H, Sekiguchi N, Sakamoto A, Sasaki S, Mamiya K, Koizumi T: Dynamics of $\mathrm{L}$-carnitine in plasma and urine in patients undergoing cisplatin chemotherapy. Chemotherapy 2017;62:225-230.

10 Berner YN, Larchian WA, Lowry SF, Nicroa RR, Brennan MF, Shike M: Low plasma carnitine in patients on prolonged total parenteral nutrition: association with low plasma lysine. JPEN J Parenter Enteral Nutr 1990;14: 255-258.

11 Hassan A, Tsuda Y, Asai A, Yokohama K, Nakamura K, Sujishi T, Ohama H, Tsuchimoto Y, Fukunishi S, Abdelaal UM, Arafa UA, Hassan AT, Kassem AM, Higuchi K: Effects of oral L-carnitine on liver functions after transarterial chemoembolization in intermediatestage HCC patients. Mediators Inflamm 2015;2015:608216.

12 Ishikawa T, Abe S, Watanabe T, Nozawa Y, Sano T, Iwanaga A, Seki K, Honma T and Yoshida $\mathrm{T}$ : L-carnitine administration to cirrhotic patients with sarcopenia improves nutritional state including controlling nutritional status (CONUT) score. Int J Nutr Sci 2016; $1: 1-3$.

13 Savica V, Santoro D, Mazzaglia G, Ciolino F, Monardo P, Calvani M, Bellinghieri G, Kopple JD: L-carnitine infusions may suppress serum C-reactive protein and improve nutritional status in maintenance hemodialysis patients. J Ren Nutr 2005;15: 225-230.

14 Muto Y, Sato S, Watanabe A, Moriwaki H, Suzuki K, Kato A, Kato M, Nakamura T, Higuchi K, Nishiguchi S, Kumada H; Long-Term Survival Study Group: Effects of oral branched-chain amino acid granules on event-free survival in patients with liver cirrhosis. Clin Gastroenterol Hepatol 2005;3: 705-713. 